\title{
SELECTION OF SUSTAINABLE CHEMICAL PROCESS DESIGN USING ANP: A BIODIESEL CASE STUDY
}

\author{
Mohamad Rizza Othman* \\ Faculty of Chemical \& Natural Resources Engineering, \\ Universiti Malaysia Pahang, Malaysia \\ E-mail: rizza@ump.edu.my \\ Günter Wozny \\ Chair of Process Dynamics and Operation \\ Berlin Institute of Technology, Germany \\ E-mail: guenter.wozny@tu-berlin.de \\ Jens-Uwe Repke \\ Institute of Thermal, Environment \& Natural Products Process Engineering \\ TU Bergakademie Freiberg, Germany \\ E-Mail: jens-uwe.repke@tun.tu-freiberg.de
}

\begin{abstract}
The design for sustainability in chemical process design should consider the three pillars of sustainability namely economic feasibility, environmental friendliness and social benefits. In reality however, agreeability among the criteria are not easily obtained because of the dependencies that exist between them. Also conflict of interest between the decision makers makes the decision making becomes more complex and therefore trade off is often needed. To encounter this complex interaction, analytic network process (ANP) is adopted utilizes its capability to account the interdependencies among level of attributes. Using ANP, a hierarchical decision network model using elements that are determinant to engineers and managers is developed that also taken into account the dependencies that exist within the framework. An example of several biodiesel process designs from literature are examined to shows the applicability of the approach. Overall, the approach offers a practical and systematic tool for aiding sustainable decision making in chemical process design specifically those that deal with complex and interacting decision environment.
\end{abstract}

Keywords: ANP, sustainability, chemical process design, biodiesel

\section{Introduction}

Depletion of non-renewable resources, global warming, forest depletion, water contamination, air pollution and many others need special attention. Nowadays, people globally believe sustainability development is very crucial since the ability of future generations meeting their needs depends on the decision is made in today's development. 'Design for sustainability' is a concept which centered around the design methodologies that balanced between the three pillars of sustainability namely economic feasibility, environmental friendliness and social benefits. As such, attaining sustainability often creates conflicting objectives which pose a multicriteria problem in decision making. Some of the decision making methodologies (MCDM) to tackle such problem are analytic hierarchy process (AHP), analytic network process (ANP), distance function method and the multi attribute utility theory (MAUT). Out of these methodologies, AHP is the most suitable and commonly use in many fields for instance in

\footnotetext{
${ }^{*}$ Corresponding author
} 
education, business, sports and military purposes (Saaty, 2008). While AHP has been very popular, ANP is less prominent in the literature. ANP, a more generalized approach, is an attractive multicriteria decision making tool because it allows for the consideration of interdependencies among and between levels of attributes (Meade and Sarkis, 1999). It is a more accurate approach for modeling complex decision especially when interactions exist in the problem environment. In engineering application there exist various researches dedicated to its application. The implementation in process system engineering (PSE) specifically in chemical process design is rather uncommon. Viewing this gap, the objective of this paper serves two fold. Firstly is to discuss the various elements that influence the decision of sustainable chemical design option and to come out with a decision problem framework. The elements within the framework not only consider the factors which are important to the technologist e.g. engineers but also to the ones that are important at the management level. Secondly its objective is to adopt the ANP framework for selection of sustainable chemical process design option. Four biodiesel process design options based on the work by Zhang et al. $(2003 \mathrm{a}, \mathrm{b})$ will be used to show the applicability of the proposed approach.

\section{Decision framework}

In the selection of process design option, the task must not made solely by the engineers' intuition. Rather it needs to take into account the reflection of other organization function as well such as the managements. Omitting them from the decision process can have detrimental organizations effects. But including them however, increases the complexity and difficulty, as sometimes-conflicting agendas and objectives must somehow be resolved (Meade \& Presley, 2002). This implied that there is a need to reconcile and integrate the needs and desires of different stakeholders in the decision environment.

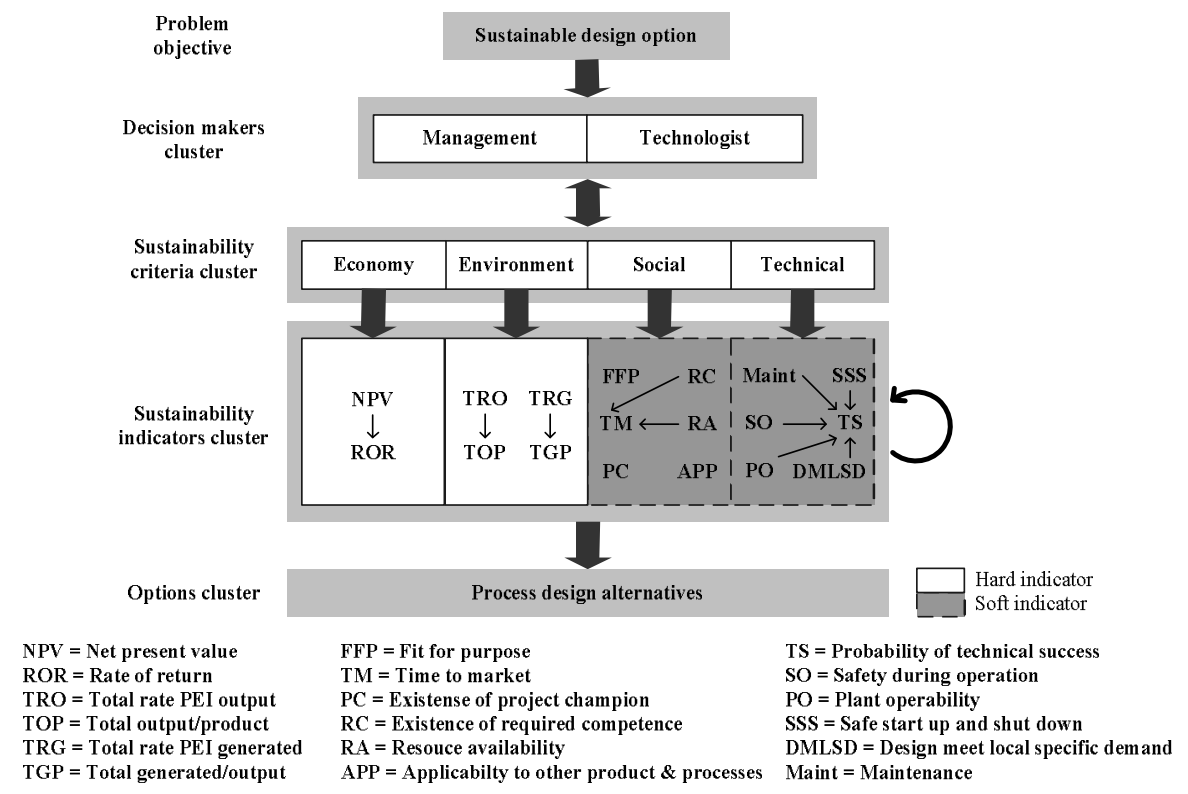

Figure 1. Decision framework based on ANP

In 1998, Herder and Weijnen conducted a study to explicitly define quality indicators for early design decision making. They observed industrial practice case studies and conducted interviews with expert panels and professionals from industry and academia. Herder and Weijnen (1998) concluded top ten quality indicators. These qualitative indicators are suitable as the guideline to assess a good quality design since they utilize the heuristics knowledge of assessors in process design evaluation. In our previous work (Othman et al., 2010) we proposed a decision framework combining quantitative and qualitative indicators to measure the system sustainability performance at early design stage. The approach utilizes 
the capability of process simulators for quantitative evaluation and human heuristics for qualitative evaluation. The approach provides a good tool to support decision making but the decision framework however is only focused within an engineer's perspective. In 2002, Meade \& Presley provide a decision framework for selection of R\&D projects from management, marketing and technologist perspective. It is in a generalized form but can be extended or adapted to meet a particular application. To take the advantage of all of these works, we embed and integrate the relevant elements and produce a new set of decision framework. The framework not only combines the technical and management requirements of a good design, it also introduces quantitative and qualitative evaluation. Also, the objective of the assessment is to evaluate a specific type of process with a few design options. But on the other hand, it also useful to assess the performance of any modified or intensified basic design. The decision framework is illustrated in Figure 1. The framework does involve representing relationships hierarchically but does not have a strict structure as does AHP. It does include a system-with-feedback where a level may both dominate and be dominated, directly or indirectly, by other decision attributes and levels (Meade and Sarkis, 1999). In general the sustainability indicators are divided into two categories; hard and soft. Hard indicators give a quantitative evaluation of process using numerical information and formulas whilst soft indicators give qualitative evaluation which depends heavily on expert's judgement that is mostly heuristic. The economic and environmental criteria and its indicators are associated with the hard category since there are quantitative methodologies to measure these indicators. On the other hand, the social and technical criteria are represented by soft-based qualitative indicators. These ill-defined indicators are very subjective because of different interpretations and often represented by specific scales as widely used in process safety engineering. In the end however, they play important roles in obtaining agreeable solutions.

\section{Case study: Biodiesel production technology}

Biodiesel is a renewable energy synthesized by alcoholysis of natural triglycerides from vegetables oil or animal fats to short chain alkyl esters. The advantage of this fuel compared to fossil fuel is that the raw materials are naturally obtainable and renewable. Added to that, the ever increasing petroleum price makes biodiesel a developing area of production and research. Although environmentally friendly, the drawback of biodiesel is the production cost which is higher than diesel fuel. Thus currently, without government subsidy, biodiesel is not economically feasible and consequently more extensive research and technological development is needed. In 2003, Zhang and his co-workers conducted a good technoeconomic assessment on four simulated biodiesel processes namely alkali-catalyzed system using virgin oil (Case 1), alkali-catalyzed system using waste cooking oil (Case 2), acid-catalyzed process using waste cooking oil (Case 3) and acid-catalyzed system using hexane extraction (Case 4). Their simulation results concluded that all of these processes proved to be technically and economically feasible though each had its limitations. Their evaluations however are limited to only techno-economic criteria and furthermore, has no selection and ranking of alternatives methodology were applied. To test the functionality of the proposed methodology the four biodiesel processes will be used as case study. As Zhang's work focuses on the techno-economic assessment additional work on environment and social assessment are performed utilizing the data and reviews included in their work. Nevertheless, the effect of energy usage towards environment was not considered.

\section{Selection of sustainable option: ANP approach}

\subsection{Problem decomposition}

The decomposed model in ANP is shown in Figure 1. The main goal of the assessment is to select the most sustainable chemical process design option that meets the sustainability criteria. The framework also contains interdependency between clusters in particular, between the decision makers cluster and sustainability criteria cluster. Other than that the framework also includes inner dependencies among the elements within the sustainability indicator cluster. It is important for the decision makers when 
considering interdependencies in ANP to carefully analyze the feedback effect of the elements in each criteria cluster. This is because they may interact or have impact or influences by some or all of the elements of that cluster or another cluster with respect to a property governing the interaction of the entire system such as energy or capital (Saaty, 1999). Taking care of this agenda is crucial to ensure that the model resembles the problem being addressed and thus, elucidate significant and convincing result.

\subsection{Pairwise comparisons and priority vectors}

Performing pairwise comparison and calculating priority vectors follows the typical procedure. However, it is important to note that assigning weights to indicators is subjective. Therefore, decision makers' knowledge, experience, and judgment ability are critical in weight assignment. Generally, in the biodiesel industry the economic feasibility is vital for its survival. Although there is increasing awareness towards environment, biodiesel production generally uses and produces non-toxic and non-pollutant materials thus its impact is relatively low. When comparing environment to social aspect, for biodiesel case, it is more important to focus on the social related issues such as safety or operability. Such consideration is necessary to ensure that the plant operates smoothly and manages to deliver timely product without jeopardizing the product quality.

\subsection{Process alternatives evaluation}

The assessment results of the four biodiesel process alternatives are shown in Table 1. The data for quantitative evaluation were obtained from Zhang's article whereas the social and technical evaluations were based on our experience using specific scale in Othman et al. (2010). It is important to note that since the unit of measurements are different, it is essential that the assessment values to be transformed into a score index. Using the approach used in Othman et al. (2010) the assessment values is transformed into score index. Then it is normalized to get a normalized score index, as shown in Table 1. Note that the transformation also takes into account the value-desirability behaviour of the indicators namely highervalue-higher-desirability (HVHD) and the lower-value-higher-desirability (LVHD). Direct comparison of the score attained by each case indicates that Case 3 is the most economically feasible while Case 2 is the least preferred. Environment performance on the other hand shows that the alkali based process (Case 1 and 2) performed better than the acid base system (case 3 and 4). For the social and technical criteria, Case 1 is assessed as the most preferred while Case 2 being the least preferred. Direct summation of the total score shows that Case 3 is the most sustainable followed by Case 1, Case 2 and Case 4. This result however is a linear comparison and that does not reflect the decision makers' preferability and interactions between the elements and could elucidate spurious and under justified answers. This is where ANP plays an important role in decision making.

\subsection{Supermatrix formation and analysis}

The next step is the formation of supermatrix. The supermatrix has similar concept to the Markov chain process (Saaty, 1996) where it allows for a resolution of the effects of interdependence that exists between the elements of the system (Meade and Sarkis, 1999). To obtain global priorities in a system with interdependent influences, the local priority vectors are entered into the appropriate columns of a matrix, known as a supermatrix. As a result, a supermatrix, $\mathbf{M}$ is actually a partitioned matrix, where each matrix segment represents a relationship between two nodes (components or clusters) in a system (Meade and Sarkis, 1999). All the pairwise comparisons priority vectors and the normalized score performed before were arranged with respect to its control criteria to form the supermatrix. Because of the presence of interdependencies, this unweighted supermatrix must be transformed to make it stochastic. This makes each column of the matrix sums to unity. A recommended approach by Saaty (1996) is to determine the relative importance of the clusters in the supermatrix with the column cluster (block) as the controlling component (Meade and Sarkis, 1999). The clusters comparison is performed and the eigenvalue obtained is used to form weighted supermatrix which is column stochastic. Raising the weighted supermatrix to powers gives the long-term relative influences of the elements on each other. The so called limit 
supermatrix has the same form as the weighted supermatrix, but all the columns of the limit supermatrix are the same. The supermatrix is raised to its limit and reaches it convergence at $\mathbf{M}^{31}$. The limit supermatrix with its stable weighted values is shown in Figure 2. The result of the alternative assessment using ANP is obtained from the alternatives block matrixes located at the bottom left of the limit supermatrix (see Figure 2). The alternative with the largest overall priority should be the one selected. According to Chung et al. (2005) since the supermatrix formed covers the whole network, the priority weights of alternatives can be found in the column of alternatives in the normalized supermatrix. On the other hand, if a supermatrix only comprises of components that are interrelated, additional calculation must be made to obtain the overall priorities of the alternatives.

Table 1. Assessment results.

\begin{tabular}{llcccccccc}
\hline Criteria & Indicators & \multicolumn{4}{c}{ Assessment value, $a$} & \multicolumn{4}{c}{ Norm. score index, $S^{N}$} \\
& & C1 & C2 & C3 & C4 & C1 & C2 & C3 & C4 \\
\hline Economy & NAP, $\left(\$ \times 10^{-6}\right)$ & -2.06 & -2.28 & -0.35 & -0.82 & 0,10 & 0,09 & 0,57 & 0,24 \\
& ROR, \% & -85.27 & -51.18 & -15.63 & -21.48 & 0,08 & 0,14 & 0,45 & 0,33 \\
Environment & TRO & 26.5 & 16.5 & 131.7 & 125.2 & 0,33 & 0,53 & 0,07 & 0,07 \\
& TOP & 0.02 & 0.01 & 0.12 & 0.11 & 0,30 & 0,60 & 0,05 & 0,05 \\
& TRG & -550 & -465 & -664 & -608 & 0,28 & 0,08 & 0,33 & 0,31 \\
& TGP & -0.49 & -0.42 & -0.59 & -0.54 & 0,24 & 0,21 & 0,29 & 0,26 \\
Social & FFP & 10 & 10 & 10 & 10 & 0,25 & 0,25 & 0,25 & 0,25 \\
& TM & 6 & 3 & 5 & 4 & 0,33 & 0,17 & 0,28 & 0,22 \\
& PC & 5 & 5 & 5 & 5 & 0,25 & 0,25 & 0,25 & 0,25 \\
& RC & 10 & 10 & 10 & 10 & 0,25 & 0,25 & 0,25 & 0,25 \\
& RA & 4 & 6 & 6 & 6 & 0,18 & 0,27 & 0,27 & 0,27 \\
& TPP & 7 & 7 & 5 & 5 & 0,29 & 0,29 & 0,21 & 0,21 \\
Technical & DMLSD & 10 & 10 & 10 & 10 & 0,25 & 0,25 & 0,25 & 0,25 \\
& TS & 10 & 10 & 10 & 10 & 0,25 & 0,25 & 0,25 & 0,25 \\
& SO & 3 & 2 & 3 & 2 & 0,30 & 0,20 & 0,30 & 0,20 \\
& OP & 5 & 1 & 5 & 3 & 0,36 & 0,07 & 0,36 & 0,21 \\
& SSS & 5 & 3 & 5 & 5 & 0,28 & 0,17 & 0,28 & 0,28 \\
& Maint & 6 & 3 & 5 & 4 & 0,33 & 0,17 & 0,28 & 0,22 \\
\hline
\end{tabular}

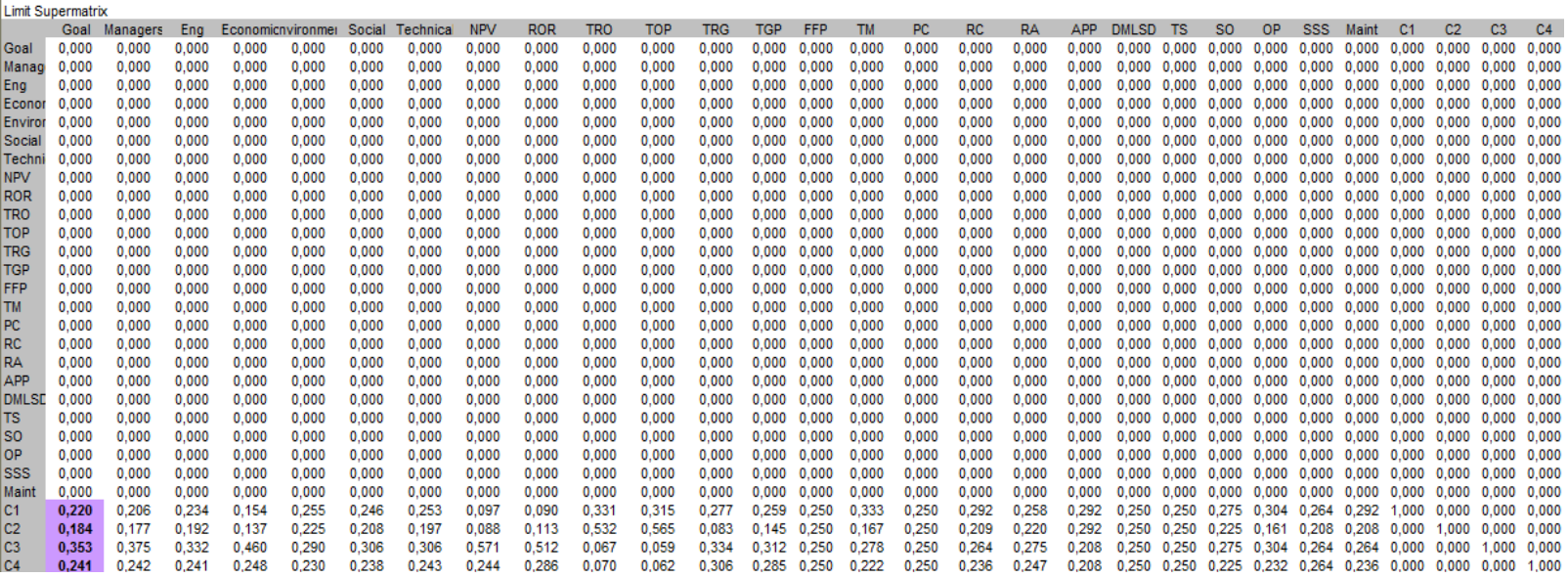

Figure 2. Results of the limit supermatrix in excel spreadsheet.

The results from Figure 2 show that the most sustainable design option is found in Case 3. It is followed by Case 4, Case 1 and finally Case 2. Overall, the alkali-catalyzed processes (Case 1 and 2) are less preferable than the acid-catalyzed systems (Case 3 and 4) mainly because of its huge economic 
disadvantages. Although the latter are environmentally unattractive, yet, the trade-off between the three other criteria makes them perform better. As a whole, the results obtained shows that the ANP is able to successfully select and rank the preferability of several design alternatives embedding the interdependencies that exists among the elements which cannot be conducted in the conventional AHP and direct comparison method.

\section{Concluding remarks}

In this work, we proposed a holistic ANP-based decision framework for selection of sustainable chemical process design option that include quantitative and qualitative factors which are determinants for both engineers and managers' perspectives. The framework however can be extended or modified depending on the specific process or decision problem environment. Other possible interdependencies can also be added depends on decision maker's intuition. The advantages of ANP rely on its structured and systematic approach. But more importantly is its account for the interdependencies among its elements. As such, ANP is capable of dealing with uncertainty and complexity in the problem environment. The approach is successfully tested to four biodiesel process technology. Compared to AHP, using ANP in cases where the elements are interacting among each other offers a more insightful and persuasive decisions. However, one drawback of ANP is the larger number of pairwise comparison that needs to be conducted compared to AHP. This number will increase with increasing complexity and interdependency. Overall, the approach is an effective decision tools for industrialists to support the selection of sustainable design option in a complex and interdependent problem environment.

\section{REFERENCES}

Chung, S.H., Lee, A.H.I., \& Pearn, W.L. (2005). Analytic network process (ANP) approach for product mix planning in semiconductor fabricator. Int. J. Production Economics, 96, 15-36.

Meade, L.M. \& Presley, A. (2002). R\&D project selection using analytic network process. IEEE Transactions on Engineering Management, 49(1), 59-66.

Meade, L.M. \& Sarkis, J. (1999). Analyzing organizational project alternatives for agile manufacturing processes: an analytic network approach. Int. J. Prod. Res., 37(2), 241-261.

Othman, M.R., Repke, J.U., Wozny, G. \& Huang, Y. (2010). A Modular Approach to Sustainability Assessment and Decision Support for Chemical Process Design. Ind. Eng. Chem. Res., 49 (17), 7870 7881 .

Saaty, T.L. (1996). Decision making with Dependence and Feedback: The Analytic Hierarchy Process. RWS Publications. Pittsburg.

Saaty, T.L. (1999). Fundamentals of The Analytic Network Process. ISAHP, Kobe, Japan, August 12-14 1999.

Saaty, T.L. (2008). Decision making with the Analytic Hierarchy Process. Int. J. Service Sciences, 1(1), 83-98.

Zhang, Y., Dub, M. A., McLean, D. D. \& Kates, M. (2003a). Biodiesel production from waste cooking oil: Process design and technological assessment. Bioresource Technology. 89, 1-16.

Zhang, Y., Dub, M. A., McLean, D. D. \& Kates, M. (2003b). Biodiesel production from waste cooking oil: Economic assessment and sensitivity analysis. Bioresource Technology. 90, 229-24. 